Supporting Information

\title{
Kinetic Study of Gas-Phase Reactions of Pyruvic Acid with $\mathrm{HO}_{2}$
}

\author{
Jonathan R. Church, Veronica Vaida, and Rex T. Skodje*
}

Department of Chemistry, University of Colorado, Boulder, CO 80309-0215

\footnotetext{
*Corresponding author: rex.skodje@colorado.edu
} 


\section{Table of Contents}

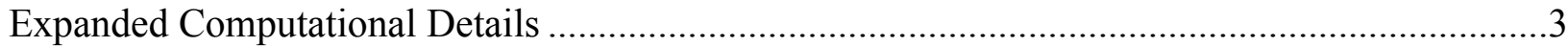

Table S.1 Cartesian Coordinates $(\AA)$ of M11/cc-pVTZ Stationary Point Structures .....................3

Table S.2 DFT energetics of reactants and intermediate structures for the $\mathrm{PA}+\mathrm{HO}_{2}$ reactions calculated using M11/cc-pVTZ structures.....................................................................14

Table S.3 DFT energetics of transition state structures of $\mathrm{PA}+\mathrm{HO}_{2}$ reactions calculated using M11/cc-pVTZ structures.

Table S.4 DFT energetics of products produced from the reactions between $\mathrm{PA}+\mathrm{HO}_{2}$ calculated

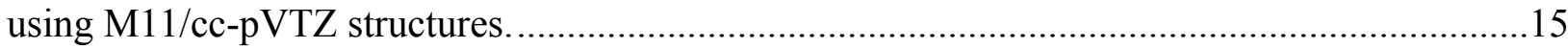

Table S.5 Ab initio energetics of reactants and transition state structures of $\mathrm{PA}+\mathrm{HO}_{2}$ reactions calculated using M11/cc-pVTZ structures..................................................................... 16 Table S.6 Harmonic frequncies of $\mathrm{PA}+\mathrm{HO}_{2}$ reactants and intermediates determined from the

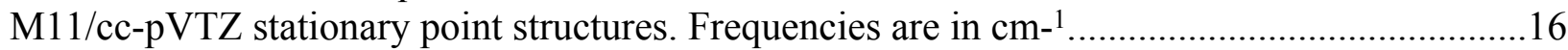




\section{Expanded Computational Details}

In this work the GAMESS-US program and the Gaussian 16 programs were used to perform all the calculations in this study. The GAMESS-US program was used to perform the geometry optimizations and saddlepoint searches using the quadratic approximation as mentioned in the main text. While searching for all stationary points, the hessian was calculated before the first geometry optimization step and then recalculated after every 10 steps. The wavefunction in the SCF calculations in both programs were treated using the restricted-open Hatree-Fock formulism.

The SCT calculations were performed using reaction path information obtained by the GS2 algorithm coded into a Python 2.7 script which worked using gradient and hessian evaluations from GAMESS-US. This code starts off using the transition state structures and a corresponding reaction frequency and then calculates the reaction path in both directions, while obtaining hessian information for SCT. A Python 2.7 script was also written for the SCT calculations which took the reaction path information and interpolated between the points before calculating the transmission coefficients.

For the kinetic rate constants, the reaction degeneracy was simply treated as the number of attack sites for each reaction, ie 3 for an abstraction of a methyl hydrogen and one for the abstraction of the hydrogen of the carboxylate group. Additionally, a chirality term was included in the rate constant if a structure had a non-superimposable mirror image. When determining the individual rate constants of $\mathrm{R} 1, \mathrm{R} 2$, and $\mathrm{R} 6$ the barriers heights were determined relative to the asymptotic energetics of the lowest energy conformer of pyruvic acid, the Tc conformer, and $\mathrm{HO}_{2}$.

\section{Table S.1 Cartesian Coordinates $(\AA)$ of M11/cc-pVTZ Stationary Point Structures}

\begin{tabular}{lrrr}
\multicolumn{4}{l}{ Pyruvic } \\
O \\
O & -7.4171756547 & 3.6308370738 & 0.0627590448 \\
$\mathrm{C}$ & -6.2854358284 & 3.9549140548 & 0.2631553398 \\
$\mathrm{C}$ & -5.5117647498 & 3.5369042851 & 1.5393389477 \\
$\mathrm{O}$ & -5.5480941741 & 4.6975046100 & -0.5527965856 \\
$\mathrm{C}$ & -6.2580159127 & 2.6988433023 & 2.5187194669 \\
$\mathrm{O}$ & -4.3729137409 & 3.9147377657 & 1.6490876477 \\
$\mathrm{H}$ & -4.6752466560 & 4.8008752618 & -0.1373159851 \\
$\mathrm{H}$ & -7.1610697064 & 3.2313080827 & 2.8265517510 \\
$\mathrm{H}$ & -5.6273333129 & 2.4662704066 & 3.3732552236 \\
$\mathrm{H}$ & -6.5988502643 & 1.7884851571 & 2.0197051491
\end{tabular}

Pyruvic Tt

$\begin{array}{llll}\mathrm{O} & -7.3212778587 & 4.2104856937 & 0.7501268297 \\ \mathrm{C} & -6.1640441834 & 3.9957019723 & 0.5190487672 \\ \mathrm{C} & -5.3123398470 & 3.0197670683 & 1.3664112083 \\ \mathrm{O} & -5.4716398584 & 4.5523679041 & -0.4717488310 \\ \mathrm{C} & -6.0598005364 & 2.3710375195 & 2.4908943046 \\ \mathrm{O} & -4.1604865818 & 2.8405797913 & 1.0965837454 \\ \mathrm{H} & -6.9159852133 & 1.8241023934 & 2.0896116383 \\ \mathrm{H} & -6.4684059302 & 3.1420265651 & 3.1479474760 \\ \mathrm{H} & -5.3937596048 & 1.7066021515 & 3.0360581594\end{array}$


$\mathrm{H} \quad-6.0651003861 \quad 5.1443389409 \quad-0.9523232979$

Pyruvic Cc

$\begin{array}{lllll}\text { O } & -7.1330219423 & 3.8162364122 & -0.1221096172\end{array}$

$\begin{array}{lllll}\text { C } & -5.8724188086 & 4.1465916412 & 0.2280533176\end{array}$

$\begin{array}{lllll}\text { C } & -5.3713519281 & 3.5738290205 & 1.5923447336\end{array}$

$\begin{array}{lllll}\text { O } & -5.1848469586 & 4.8291986954 & -0.4553376262\end{array}$

$\begin{array}{lllll}\text { C } & -6.3118089836 & 2.7116598217 & 2.4000325361\end{array}$

$\begin{array}{lllll}\mathrm{O} & -4.2616603030 & 3.8436234713 & 1.9387520766\end{array}$

$\mathrm{H} \quad \begin{array}{lllll}\mathrm{H} & -7.5546688710 & 3.2594105161 & 0.5374279763\end{array}$

$\begin{array}{lllll}\mathrm{H} & -7.2158222897 & 3.2709661930 & 2.6609141292\end{array}$

$\mathrm{H} \quad-\quad-5.8084555323 \quad 2.4001087388 \quad 3.3124301334$

$\begin{array}{llll}\mathrm{H} & -6.6036633629 & 1.8244909997 & 1.8291785806\end{array}$

Pyruvic Ct

$\begin{array}{llll}\mathrm{O} & -7.1686921281 & 3.8230098334 & 0.0101844176 \\ \mathrm{C} & -5.8886046131 & 4.1678257323 & 0.2498999023 \\ \mathrm{C} & -5.3760045428 & 3.5715998180 & 1.5894811171 \\ \mathrm{O} & -5.2300196029 & 4.8457898309 & -0.4744899236 \\ \mathrm{C} & -6.3394562539 & 2.7386778267 & 2.3828453269 \\ \mathrm{O} & -4.2491219707 & 3.8098956616 & 1.9085209031 \\ \mathrm{H} & -7.4302722976 & 4.2205139890 & -0.8308661919 \\ \mathrm{H} & -7.2262236649 & 3.3298390252 & 2.6224314270 \\ \mathrm{H} & -5.8515725266 & 2.3900686596 & 3.2902146504 \\ \mathrm{H} & -6.6806737994 & 1.8952388734 & 1.7782856211\end{array}$

$\mathrm{HO} 2$

$\begin{array}{lllll}\mathrm{O} & -7.9326186490 & 4.1390193675 & -0.0142449704\end{array}$

$\begin{array}{lllll}\mathrm{O} & -6.6273235565 & 4.2092115394 & 0.0248247072\end{array}$

$\begin{array}{lllll}\mathrm{H} & -8.2560377945 & 4.8233890931 & 0.5936802632\end{array}$

$\mathrm{OH}$

$\begin{array}{lllll}\mathrm{H} & -10.0538643661 & 4.4156700000 & 0.0000000000\end{array}$

$\begin{array}{lllll}\mathrm{O} & -9.0822356339 & 4.4156700000 & 0.0000000000\end{array}$

$\begin{array}{lrrr}\mathrm{H} 2 \mathrm{O} 2 & & & \\ \mathrm{H} & -3.1350235978 & 4.8236904559 & 1.0958536006 \\ \mathrm{O} & -3.2285273478 & 3.3100182073 & -0.0003463994 \\ \mathrm{O} & -2.6800326657 & 4.5918793347 & 0.2792744727 \\ \mathrm{H} & -3.7270863888 & 3.4838120021 & -0.8058116739\end{array}$

$\mathrm{CO} 2$

$\begin{array}{lllll}\text { C } & -8.7514965771 & 5.2097140269 & 0.0000000000\end{array}$

$\begin{array}{lllll}\mathrm{O} & -7.6025773059 & 5.0832787365 & 0.0000000000\end{array}$

$\begin{array}{lllll}\mathrm{O} & -9.9004161169 & 5.3361472366 & -0.0000000000\end{array}$

PRC1

$\begin{array}{llll}\text { C } & 1.0182257117 & -0.1860631366 & 0.0109279017\end{array}$

$\begin{array}{lllll}\text { C } & -0.5303120540 & -0.1131414786 & 0.0372609459\end{array}$

$\begin{array}{llll}\text { O } & 1.6209274816 & -0.3556830586 & 1.0313646783\end{array}$ 


$\begin{array}{lrrr}\mathrm{O} & 1.5731701605 & -0.0511573729 & -1.1735630168 \\ \mathrm{O} & -1.1432063781 & 0.0585951579 & -0.9901733202 \\ \mathrm{C} & -1.1572863784 & -0.2666516215 & 1.3778682463 \\ \mathrm{H} & -0.7596945020 & 0.5029409136 & 2.0438118785 \\ \mathrm{H} & -2.2396241891 & -0.2026334073 & 1.2981867120 \\ \mathrm{H} & -1.3524952009 & 0.3078710804 & -2.7382449266 \\ \mathrm{H} & -0.8441975049 & -1.2215317429 & 1.8068418054 \\ \mathrm{O} & -1.0341405832 & 0.4177264051 & -3.6604398842 \\ \mathrm{O} & 0.2605263002 & 0.3412054479 & -3.5751602929 \\ \mathrm{H} & 0.9284418927 & 0.0803981193 & -1.9027970599 \\ \mathrm{PRC} 2 & & & \\ \mathrm{O} & -7.4222150781 & 4.4039064839 & 0.1793505565 \\ \mathrm{C} & -6.2402516885 & 4.3618765529 & 0.3329106966 \\ \mathrm{C} & -5.5856290400 & 3.4527091212 & 1.4030783837 \\ \mathrm{O} & -5.3544103337 & 5.0608212646 & -0.3641268094 \\ \mathrm{C} & -6.4994953858 & 2.6323378578 & 2.2317480220 \\ \mathrm{O} & -4.3759132595 & 3.4811309050 & 1.4818453891 \\ \mathrm{H} & -7.1011061750 & 2.0057842950 & 1.5677322406 \\ \mathrm{H} & -7.2079894741 & 3.2991946612 & 2.7305137358 \\ \mathrm{H} & -5.9363249550 & 2.0362656224 & 2.9471891305 \\ \mathrm{O} & -2.8673879882 & 1.9933744869 & 3.2922143887 \\ \mathrm{O} & -3.8419573201 & 1.3767837526 & 3.9036995228 \\ \mathrm{H} & -3.3012857043 & 2.5575041523 & 2.6161217300 \\ \mathrm{H} & -4.4684130576 & 4.8384323241 & -0.0367594968\end{array}$

PRC3

$\mathrm{O}$

C

$\begin{array}{ccc}-7.5552641311 & 4.3453914215 & 0.2365490832 \\ -6.3376296029 & 4.3410898364 & 0.3026870407 \\ -5.6091758807 & 3.4581325072 & 1.3466411268 \\ -5.5435591462 & 5.0394894329 & -0.4541379654 \\ -6.5152201046 & 2.6504032834 & 2.2236219687 \\ -4.4147791794 & 3.4700086367 & 1.3924802228 \\ -7.1417156867 & 2.0044916189 & 1.6043657544 \\ -7.1939234689 & 3.3178482413 & 2.7595513294 \\ -5.9192320684 & 2.0635565156 & 2.9182837959 \\ -7.3172198120 & 6.3363031667 & -2.0242762825 \\ -8.4929644312 & 5.9439036111 & -1.6355882442 \\ -8.3189650726 & 5.2986131568 & -0.8900093507 \\ -6.0861536152 & 5.5751153414 & -1.0928471692\end{array}$

PRC4

$\mathrm{O}$

$\begin{array}{ccc}-7.4712805362 & 4.3292008687 & 0.1970927262 \\ -6.2803446548 & 4.2991128153 & 0.3206798030 \\ -5.5852597219 & 3.4284344025 & 1.3927297378 \\ -5.4135992420 & 4.9842535563 & -0.4180033652 \\ -6.5052561055 & 2.6305728485 & 2.2490616382 \\ -4.3833635456 & 3.4469655338 & 1.4689718340 \\ -7.1125633091 & 1.9849483390 & 1.6095798240 \\ -7.2058072593 & 3.3086314451 & 2.7430329262 \\ -5.9336773881 & 2.0518001094 & 2.9721407091 \\ -2.8687838547 & 2.0126803421 & 3.2730546236\end{array}$ 


$\begin{array}{crrr}\mathrm{O} & -3.8269482799 & 1.4024403212 & 3.9155855276 \\ \mathrm{H} & -3.3203192217 & 2.5612065000 & 2.5923041282 \\ \mathrm{H} & -5.9116059012 & 5.5035297782 & -1.0633250227 \\ & & & \\ \mathrm{INT} 1 & & & \\ \mathrm{O} & -7.3581444454 & 3.9205070385 & 0.2449362862 \\ \mathrm{C} & -6.1742306212 & 3.9556428156 & 0.4388492862 \\ \mathrm{C} & -5.4723998558 & 3.1330229336 & 1.4783001807 \\ \mathrm{O} & -5.3367532114 & 4.7379020264 & -0.2537995329 \\ \mathrm{C} & -6.1776079970 & 2.3122154286 & 2.2411556364 \\ \mathrm{O} & -4.1338586086 & 3.3352047442 & 1.5179461352 \\ \mathrm{H} & -7.2468707051 & 2.2583370627 & 2.0913760513 \\ \mathrm{H} & -5.7120927781 & 1.6993480878 & 3.0044590853 \\ \mathrm{H} & -3.7423496085 & 2.7833696779 & 2.1996163160 \\ \mathrm{H} & -5.8708594488 & 5.2258894047 & -0.8932587443 \\ & & & \\ \mathrm{INT} 2 & & & \\ \mathrm{C} & 0.7782804957 & -0.0756797878 & -0.0326958674 \\ \mathrm{C} & -0.7336864045 & -0.0566499911 & 0.0195789666 \\ \mathrm{O} & 1.3549554916 & 1.1134217912 & -0.2960965503 \\ \mathrm{H} & 0.6911401296 & 1.7520347034 & -0.5733493174 \\ \mathrm{O} & 1.4178887599 & -1.0593873947 & 0.1625484444 \\ \mathrm{O} & -1.2420675211 & -1.2650353532 & -0.2830107394 \\ \mathrm{C} & -1.4271235279 & 1.0181260309 & 0.3779604511 \\ \mathrm{H} & -2.5087713307 & 0.9957704976 & 0.4478004658 \\ \mathrm{H} & -0.9273961614 & 1.9305711172 & 0.6741693375 \\ \mathrm{H} & -2.1931987914 & -1.2715796536 & -0.1461536009\end{array}$

INT3

$\begin{array}{lrrr}\mathrm{C} & 2.2413284834 & 0.0719081451 & -0.0753370014 \\ \mathrm{C} & -1.2837530044 & 0.4355687740 & -0.8612944247 \\ \mathrm{O} & 3.2414375969 & 0.3794697610 & -0.5570947178 \\ \mathrm{H} & -0.5604430023 & 1.4529986995 & -2.0221839612 \\ \mathrm{O} & 1.2551012819 & -0.2689501464 & 0.4322217940 \\ \mathrm{O} & -1.4558128638 & -0.8402344422 & -0.9602831750 \\ \mathrm{C} & -1.5517459903 & 0.9988207653 & 0.4817083599 \\ \mathrm{H} & -1.7475046380 & 0.2552291942 & 1.2660204186 \\ \mathrm{H} & -0.6991608121 & 1.6261981438 & 0.7539683659 \\ \mathrm{H} & -1.7024718749 & -1.2664608773 & -0.1184176026 \\ \mathrm{O} & 0.1104947846 & 2.1428886200 & -2.4049076808 \\ \mathrm{O} & 1.0268049194 & 2.1983372141 & -1.4802912133 \\ \mathrm{H} & -2.4083208704 & 1.6745638788 & 0.3826685884\end{array}$

INT4

$\begin{array}{llll}\mathrm{C} & 2.0380355070 & -0.0523470640 & 0.0073969866\end{array}$

$\begin{array}{lllll}\text { C } & -1.1029660709 & -0.0984904006 & -0.5190569027\end{array}$

$\begin{array}{lllll}\mathrm{O} & & 1.9229929942 & 1.0397816195 & 0.3699831065\end{array}$

$\begin{array}{lllll}\mathrm{O} & 2.1464393870 & -1.1474327273 & -0.3456379098\end{array}$

$\begin{array}{lllll}\mathrm{O} & -2.1906706879 & 0.3872571226 & -1.0086035575\end{array}$

$\begin{array}{lllll}\text { C } & -1.2493374479 & -0.4881954134 & 0.9021786603\end{array}$ 


$\begin{array}{lrrr}\mathrm{H} & -0.6478603086 & 0.2254844619 & 1.4793356099 \\ \mathrm{H} & -2.2818632585 & -0.4696692705 & 1.2568136152 \\ \mathrm{H} & -1.9876506194 & 0.6398644458 & -1.9443596501 \\ \mathrm{H} & -0.7884655191 & -1.4667817559 & 1.0579137456 \\ \mathrm{O} & -0.7680559801 & 0.7138820613 & -3.2462761562 \\ \mathrm{O} & 0.2973816250 & 0.1593913583 & -2.7380628818 \\ \mathrm{H} & -0.0209051307 & -0.0722553979 & -1.7507115361\end{array}$

INT5

C

INT6

$\begin{array}{lllll}\text { C } & 1.2607219121 & 0.0364585129 & -0.3198325945\end{array}$

$\begin{array}{lllll}\mathrm{C} & 0.0252224498 & 0.3388275886 & 0.5495027684\end{array}$

$\begin{array}{lllll}\mathrm{C} & 0.3681501930 & 0.4398572894 & 2.0074784951\end{array}$

$\begin{array}{llll}\text { O } & -0.8102744879 & -0.8917885895 & 0.4164732765\end{array}$

$\begin{array}{lllll}\mathrm{O} & & -0.6734064913 & 1.4234904026 & 0.1107775427\end{array}$

$\begin{array}{lllll}\mathrm{O} & -1.4699679191 & -0.8884241608 & -0.6948505427\end{array}$

$\begin{array}{lllll}\text { O } & 2.2719077709 & -0.4508242005 & 0.0892618265\end{array}$

$\begin{array}{lllll}\mathrm{O} & 1.0464150714 & 0.3712162564 & -1.6000403152\end{array}$

$\begin{array}{lllll}\mathrm{H} & -0.8818109309 & 1.2976555538 & -0.8239223450\end{array}$

$\begin{array}{lllll}\mathrm{H} & 0.8643183707 & -0.4745688166 & 2.3291957773\end{array}$

$\begin{array}{lllll}\mathrm{H} & -0.5515595959 & 0.5946826048 & 2.5704841017\end{array}$

$\begin{array}{lllll}\mathrm{H} & 1.0408704561 & 1.2833636260 & 2.1601816993\end{array}$

$\begin{array}{lllll}\mathrm{H} & 1.8248232512 & 0.1227888629 & -2.1153957599\end{array}$

$\begin{array}{lrrr}\text { INT7 } & & & \\ \text { C } & 1.4894880001 & 0.2171679747 & -0.3830726120 \\ \text { C } & 0.1435586371 & 0.3639436716 & 0.3399142301 \\ \text { C } & 0.2904595325 & 0.3363894809 & 1.8410318373 \\ \text { O } & -0.6146111929 & -0.8475902583 & -0.0681331609 \\ \text { O } & -0.4875359971 & 1.4771316221 & -0.1538564276 \\ \text { O } & -1.8615392360 & -0.7015942162 & 0.2345548221 \\ \text { O } & 2.5375931533 & 0.1146586873 & 0.1845056194 \\ \text { O } & 1.3357053030 & 0.2160311478 & -1.7052554417 \\ \text { H } & -1.3686438555 & 1.5170920988 & 0.2367847191 \\ \text { H } & 0.8082412175 & -0.5695110619 & 2.1541298600 \\ \text { H } & -0.7033659336 & 0.3692483548 & 2.2915140046 \\ \text { H } & 0.8722994350 & 1.2025538177 & 2.1541852996\end{array}$




$\begin{array}{lrcc}\mathrm{H} & 2.2073996166 & 0.1176176806 & -2.1100019399 \\ \mathrm{INT} 8 & & & \\ \mathrm{C} & 1.4763835120 & 0.0146417060 & -0.3395987328 \\ \mathrm{C} & 0.1407472556 & 0.2755552469 & 0.3868917248 \\ \mathrm{C} & 0.3059732049 & 0.4657821295 & 1.8731484666 \\ \mathrm{O} & -0.6146941423 & -0.9854424986 & 0.1730962469 \\ \mathrm{O} & -0.5273863293 & 1.2988946292 & -0.2563492902 \\ \mathrm{O} & -1.8693355862 & -0.7875880556 & 0.4037986344 \\ \mathrm{O} & 2.3232044417 & -0.6681758278 & 0.1467212185 \\ \mathrm{O} & 1.5743659124 & 0.5905606835 & -1.5337747463 \\ \mathrm{H} & -1.4469086005 & 1.2929950467 & 0.0398125708 \\ \mathrm{H} & 0.8794020321 & -0.3649703971 & 2.2822730511 \\ \mathrm{H} & -0.6816092789 & 0.5053119900 & 2.3373170465 \\ \mathrm{H} & 0.8363898777 & 1.3998934955 & 2.0589127458 \\ \mathrm{H} & 0.7754429407 & 1.1120545818 & -1.6997820161\end{array}$

INT9

C $\quad-1.0657607238-0.7954689998 \quad-0.3620608242$

$\mathrm{H} \quad-0.6489300971-1.7385000312 \quad-0.7336342443$

$\begin{array}{lllll}\mathrm{C} & & -0.0061688422 & 0.2497657413 & -0.3289785731\end{array}$

$\begin{array}{lllll}\text { O } & 0.9289933489 & -0.0112464316 & 0.6759436766\end{array}$

$\begin{array}{lllll}\mathrm{O} & 1.9500498315 & 0.9727766272 & 0.6051998429\end{array}$

O $\quad 0.5296911567 \quad 0.5694759530 \quad-1.5314600955$

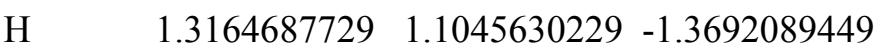

$\begin{array}{llll}\text { C } & -0.7700380059 & 1.4406515134 & 2.5362512559\end{array}$

$\begin{array}{lllll}\text { O } & 0.1073026293 & 2.1972872700 & 2.4797538789\end{array}$

$\begin{array}{llll}\mathrm{O} & -1.6529328008 & 0.7046847113 & 2.6158144875\end{array}$

$\mathrm{H} \quad \begin{array}{llll}1.6469892288 & 1.6242381926 & 1.2544600182\end{array}$

$\mathrm{H} \quad \begin{array}{lllll}1.8598398545 & -0.4765795840 & -1.0366252803\end{array}$

$\mathrm{H} \quad \begin{array}{lllll}\mathrm{H} & -1.4779394137 & -0.9545459751 & 0.6330820823\end{array}$

$\begin{array}{lrrr}\text { INT10 } & & & \\ \text { C } & -1.0548298553 & -0.7830704865 & -0.3496966800 \\ \text { H } & -0.6870348984 & -1.7526908785 & -0.7053025104 \\ \text { C } & 0.0411425840 & 0.2211631894 & -0.3475962849 \\ \text { O } & 1.0022233769 & -0.0677291939 & 0.6069978216 \\ \text { O } & 1.9272382309 & 1.0159391500 & 0.6415943954 \\ \text { O } & 0.5319274062 & 0.5621010477 & -1.5678255696 \\ \text { H } & 1.3121518903 & 1.1113331544 & -1.4272582033 \\ \text { H } & 1.5419811719 & 1.5879811592 & 1.3179191595 \\ \text { H } & -1.8464844427 & -0.4466387190 & -1.0184645107 \\ \text { H } & -1.4547619439 & -0.9039099228 & 0.6563500623 \\ & & & \\ \text { P1 } & & & \\ \text { O } & -4.4274147983 & 3.4525541219 & 0.0072643842 \\ \text { C } & -4.5163876175 & 3.5849124296 & 1.1915500595 \\ \text { C } & -5.8328172060 & 3.3606856649 & 1.9662387288 \\ \text { O } & -3.5217732172 & 3.9353996519 & 1.9952186490 \\ \text { C } & -6.9732478899 & 2.9798937208 & 1.2045003813 \\ \text { O } & -5.8180165737 & 3.5221313915 & 3.1779228632\end{array}$




$\begin{array}{llll}\mathrm{H} & -3.8803520786 & 3.9644932557 & 2.8989841125 \\ \mathrm{H} & -7.9116840290 & 2.8147720136 & 1.7167022323 \\ \mathrm{H} & -6.8846544898 & 2.8623830600 & 0.1329257493 \\ & & & \\ \mathrm{P} 2 & & & \\ \mathrm{C} & -5.5300596636 & 3.7870603101 & 1.7928104515 \\ \mathrm{C} & -6.1919123287 & 2.5829360226 & 2.4184245944 \\ \mathrm{O} & -4.6675970000 & 4.4551890585 & 2.2250190403 \\ \mathrm{H} & -7.2599393053 & 2.7852822802 & 2.5044237391 \\ \mathrm{H} & -5.7555407324 & 2.3701258364 & 3.3962109731 \\ \mathrm{H} & -6.0685809699 & 1.7350464921 & 1.7440012016\end{array}$

P6

$\begin{array}{llll}\text { C } & 1.0680562406 & -0.9031861628 & -0.0000027826\end{array}$

$\begin{array}{lllll}\mathrm{C} & & 0.0013001752 & 0.1518289183 & 0.0000071864\end{array}$

$\begin{array}{lllll}\mathrm{O} & & 0.1790414513 & 1.3379488951 & 0.0000517217\end{array}$

$\begin{array}{lllll}\mathrm{O} & -1.2379694048 & -0.3877730531 & -0.0000348977\end{array}$

$\mathrm{H} \quad 2.0456119185 \quad-0.4287541113-0.0000372460$

$\mathrm{H} \quad \begin{array}{llll}\mathrm{H} & 0.9501435908 & -1.5358657563 & 0.8807856189\end{array}$

$\mathrm{H} \quad 0.9500951527-1.5359135428-0.8807505198$

$\mathrm{H} \quad \begin{array}{lllll} & -1.8670501243 & 0.3446538129 & -0.0000190808\end{array}$

TS1

$\begin{array}{lllll}\mathrm{O} & -4.3224488055 & 2.9643504776 & 0.2706314156\end{array}$

$\begin{array}{lllll}\text { C } & -4.5106756697 & 3.4226383402 & 1.3568721930\end{array}$

$\begin{array}{lllll}\text { C } & -5.8988957059 & 3.4174001283 & 2.0368785366\end{array}$

$\begin{array}{lllll}\text { O } & -3.5841694255 & 3.9899554433 & 2.1173310836\end{array}$

$\begin{array}{llll}\text { C } & -6.9726762627 & 2.7519663155 & 1.3268378311\end{array}$

$\begin{array}{lllll}\text { O } & -5.9904248592 & 3.9018294088 & 3.1492493593\end{array}$

$\begin{array}{lllll}\mathrm{H} & -4.0175129446 & 4.2682167157 & 2.9413917809\end{array}$

$\begin{array}{llll}\mathrm{H} & -7.9781697602 & 3.0070348025 & 1.6436925513\end{array}$

$\mathrm{H} \quad \begin{array}{llll}-6.8219435582 & 1.5300872563 & 1.9320798195\end{array}$

$\mathrm{H} \quad \begin{array}{llll}\mathrm{H} & -6.7982986691 & 2.5237523950 & 0.2810540183\end{array}$

$\begin{array}{lllll}\mathrm{O} & -6.6515537174 & 0.6519149373 & 2.6710375621\end{array}$

$\begin{array}{lllll}\text { O } & -5.5288797637 & 0.9940015339 & 3.3696143849\end{array}$

$\mathrm{H} \quad \begin{array}{llll}-5.8665600883 & 1.4242263354 & 4.1684913738\end{array}$

TS2

$\begin{array}{lllll}\mathrm{O} & -7.4474078260 & 4.3397033262 & 0.4923062883\end{array}$

$\begin{array}{lllll}\text { C } & -6.2695944065 & 4.2044243547 & 0.2832263193\end{array}$

$\begin{array}{lllll}\text { C } & & -5.5265082031 & 3.7532469895 & 1.7206655914\end{array}$

$\begin{array}{lllll}\text { O } & -5.5057735259 & 4.3783467189 & -0.6819466911\end{array}$

$\begin{array}{lllll}\text { C } & -6.2001781863 & 2.6159761624 & 2.4216434935\end{array}$

$\begin{array}{lllll}\text { O } & -4.6559251509 & 4.4352510976 & 2.1662369080\end{array}$

$\mathrm{H} \quad \begin{array}{llll}-3.4391917156 & 4.9944504682 & 0.8809366632\end{array}$

$\begin{array}{lllll}\text { O } & -3.3153350079 & 3.4407459539 & -0.1413690222\end{array}$

$\begin{array}{lllll}\mathrm{O} & & -2.7293721214 & 4.5383726960 & 0.3748577461\end{array}$

$\mathrm{H} \quad \begin{array}{lllll}\mathrm{H} & -4.2756912391 & 3.7809924885 & -0.4468370968\end{array}$

$\begin{array}{lllll}\mathrm{H} & -7.2612577805 & 2.8546051222 & 2.5014276498\end{array}$

$\begin{array}{llll}\mathrm{H} & -5.7438704777 & 2.4601925551 & 3.3970478538\end{array}$ 


$\begin{array}{lccc}\mathrm{H} & -6.1067350892 & 1.7230118269 & 1.7992043466 \\ \mathrm{TS} 3 & & & \\ \mathrm{O} & -7.3531525411 & 4.0253250235 & 0.3667697736 \\ \mathrm{C} & -6.1600950597 & 3.9907768424 & 0.4436699628 \\ \mathrm{C} & -5.4335131874 & 3.1674947418 & 1.5038365401 \\ \mathrm{O} & -5.3182113365 & 4.6423177622 & -0.3528883222 \\ \mathrm{C} & -6.1649399270 & 2.3453353142 & 2.3619482257 \\ \mathrm{O} & -4.1834208817 & 3.3601736914 & 1.5995828896 \\ \mathrm{H} & -7.2338299102 & 2.3193716945 & 2.1990811390 \\ \mathrm{H} & -5.7026134675 & 2.8193410679 & 3.5043839833 \\ \mathrm{H} & -5.6849562731 & 1.3896306407 & 2.5759140427 \\ \mathrm{O} & -3.6705819014 & 2.5845304905 & 3.8605909458 \\ \mathrm{O} & -4.7586564700 & 2.9681961387 & 4.4508235760 \\ \mathrm{H} & -3.8345200818 & 2.8978452002 & 2.5385092974 \\ \mathrm{H} & -5.8381786425 & 5.1358116222 & -1.0010819239\end{array}$

TS4

$\begin{array}{lllll}\text { C } & 0.8299703649 & 0.0215799518 & 0.0359153113\end{array}$

$\begin{array}{lllll}\text { C } & -0.7075821396 & -0.0298404919 & 0.0042683982\end{array}$

$\begin{array}{llllll}\mathrm{O} & & 1.3394394070 & 1.0342101600 & -0.5379028122\end{array}$

$\begin{array}{lllll}\mathrm{H} & 0.6493067159 & 1.7524026417 & -1.2914975022\end{array}$

$\begin{array}{lllll}\mathrm{O} & 1.3406991698 & -0.8776445300 & 0.6595488183\end{array}$

$\begin{array}{lllll}\text { O } & -1.2073529578 & -1.1032293902 & -0.5092518478\end{array}$

$\begin{array}{lllll}\text { C } & -1.4715768337 & 1.0487808244 & 0.4659119311\end{array}$

$\begin{array}{lllll}\mathrm{H} & -2.5284454489 & 0.8699874476 & 0.6541189035\end{array}$

$\begin{array}{lllll}\mathrm{H} & -0.9632060183 & 1.6656503003 & 1.2034335633\end{array}$

$\mathrm{H} \quad-2.1720281867 \quad-1.1357285310-0.4394218130$

$\begin{array}{lllll}\mathrm{O} & 0.0778192437 & 2.4475776560 & -2.0486785080\end{array}$

$\begin{array}{lllll}\mathrm{O} & -1.1480549002 & 2.6363258891 & -1.6810364466\end{array}$

$\begin{array}{lllll}\mathrm{H} & -1.3733706060 & 1.7827460820 & -0.5431350558\end{array}$

TS5

$\begin{array}{llll}\text { C } & 1.5648055819 & -0.1959505781 & -0.1155360310\end{array}$

$\begin{array}{lllll}\text { C } & -0.8719081617 & -0.0801656807 & -0.0820428792\end{array}$

$\begin{array}{lllll}\text { O } & 1.6525963928 & -0.3566258451 & 1.0292032042\end{array}$

$\begin{array}{lllll}\mathrm{O} & 1.8234794806 & -0.0535862855 & -1.2518431676\end{array}$

$\begin{array}{llllll}\mathrm{O} & & -1.8586611783 & 0.0803753719 & -0.8952512782\end{array}$

$\begin{array}{lllll}\text { C } & -1.3487437993 & -0.2481927261 & 1.3138110696\end{array}$

$\begin{array}{lllll}\mathrm{H} & & -0.8647533243 & 0.5207191640 & 1.9246169793\end{array}$

$\begin{array}{lllll}\mathrm{H} & -2.4347567367 & -0.2106789661 & 1.4210802579\end{array}$

$\begin{array}{lllll}\mathrm{H} & & -1.5205894975 & 0.1898084304 & -1.8134907526\end{array}$

$\begin{array}{lllll}\mathrm{H} & -0.9468538332 & -1.1942338516 & 1.6905792237\end{array}$

$\begin{array}{lllll}\mathrm{O} & -0.8260755482 & 0.3717982555 & -3.3953259090\end{array}$

$\begin{array}{llllll}\mathrm{O} & & 0.4576602657 & 0.3319158570 & -3.5786048339\end{array}$

$\begin{array}{llllll}\mathrm{H} & & 0.8824635483 & 0.1905758344 & -2.6998929333\end{array}$

TS6

$\begin{array}{lllll}\text { C } & -7.7766910874 & 4.1057179926 & 0.6675813481\end{array}$

$\begin{array}{lllll}\text { C } & -6.3284879729 & 3.7887065731 & 0.6175974982\end{array}$ 


$\begin{array}{llll}\mathrm{C} & -5.4280344295 & 3.8743822717 & 1.7777770502 \\ \mathrm{O} & -5.8704682367 & 3.3408475204 & -0.5100598855 \\ \mathrm{O} & -5.9520237420 & 2.7787108002 & 2.1076384133 \\ \mathrm{O} & -4.5189582030 & 4.5741692409 & 2.1825354450 \\ \mathrm{H} & -3.3330191881 & 4.8100114738 & 1.1185978036 \\ \mathrm{O} & -3.2376999110 & 3.9311706753 & -0.5040733174 \\ \mathrm{O} & -2.6868786375 & 4.7329835138 & 0.3517845911 \\ \mathrm{H} & -4.8829553681 & 3.4144544917 & -0.5340374351 \\ \mathrm{H} & -7.9401225707 & 5.0165091347 & 0.0856310931 \\ \mathrm{H} & -8.1073487451 & 4.2573207310 & 1.6916753540 \\ \mathrm{H} & -8.3384211079 & 3.2956794307 & 0.2002560614\end{array}$

$\begin{array}{lrrr}\text { TS7 } & & & \\ \text { C } & 1.3878345547 & 0.0821748342 & -0.3145713851 \\ \text { C } & 0.1814583541 & 0.5222107316 & 0.5222440893 \\ \text { C } & 0.3648337495 & 0.4129991603 & 2.0016984893 \\ \text { O } & -0.8103758639 & -1.0652387105 & 0.1464262578 \\ \text { O } & -0.6305859822 & 1.3453105257 & 0.0046388154 \\ \text { O } & -1.7725168924 & -0.5846249323 & -0.5175669707 \\ \text { O } & 2.4110175081 & -0.3186838220 & 0.1616326188 \\ \text { O } & 1.1489807530 & 0.1935384924 & -1.6228750548 \\ \text { H } & -1.4533228562 & 0.5673479254 & -0.4750908202 \\ \text { H } & 0.8023439717 & -0.5495154146 & 2.2632535469 \\ \text { H } & -0.5945537367 & 0.5595439681 & 2.4952746535 \\ \text { H } & 1.0580815145 & 1.1965297153 & 2.3184898596 \\ \text { H } & 1.9300167858 & -0.1190800236 & -2.0973233299\end{array}$

$\begin{array}{lrrr}\text { TS } 8 & & & \\ \text { C } & 1.3924152320 & 0.0013984317 & -0.3285278025 \\ \text { C } & 0.1701135176 & 0.4481942552 & 0.4963489621 \\ \text { C } & 0.3450352423 & 0.4431487303 & 1.9801939501 \\ \text { O } & -0.8117862694 & -1.1220869128 & 0.2813815597 \\ \text { O } & -0.6400138304 & 1.2478236341 & -0.0887644008 \\ \text { O } & -1.8723047403 & -0.6902276759 & -0.2579725759 \\ \text { O } & 2.2682797376 & -0.6665807590 & 0.1290855044 \\ \text { O } & 1.3486763985 & 0.4336680670 & -1.5858995611 \\ \text { H } & -1.5758763015 & 0.4436387988 & -0.3265385212 \\ \text { H } & 0.8485276119 & -0.4697452599 & 2.2946859232 \\ \text { H } & -0.6235612151 & 0.5509316013 & 2.4672220990 \\ \text { H } & 0.9751061522 & 1.2954733904 & 2.2476172462 \\ \text { H } & 0.5663100445 & 1.0012918490 & -1.6751745531\end{array}$

TS9

$\begin{array}{lllll}\mathrm{C} & 1.3507879706 & 0.0514110422 & -0.2402875373\end{array}$

$\begin{array}{lllll}\mathrm{C} & & 0.1255121371 & 0.4871566041 & 0.5706450032\end{array}$

$\begin{array}{lllll}\mathrm{O} & 0.3041257974 & 0.4257251612 & 1.8752128955\end{array}$

$\begin{array}{lllll}\text { O } & -0.8525381599 & -1.1285198940 & 0.1600348982\end{array}$

$\begin{array}{lllll}\mathrm{O} & & -0.7366002636 & 1.2854345944 & 0.0798009323\end{array}$

$\begin{array}{llll}\mathrm{O} & -1.9282227760 & -0.6612917311 & -0.3207413430\end{array}$

$\begin{array}{lllll}\text { O } & 2.2791633258 & -0.4066683186 & 0.3754450189\end{array}$

$\begin{array}{lllll}\text { C } & 1.2682490934 & 0.2189836986 & -1.7195923056\end{array}$ 


$\begin{array}{lrrr}\mathrm{H} & -1.5802187280 & 0.5834910881 & -0.2350013724 \\ \mathrm{H} & 2.1609264018 & -0.1846761119 & -2.1909681025 \\ \mathrm{H} & 1.1516273531 & 1.2806332806 & -1.9503214730 \\ \mathrm{H} & 0.3721178369 & -0.2864516730 & -2.0888453450 \\ \mathrm{H} & 1.1414501313 & -0.0463977806 & 2.0274785807\end{array}$

$\begin{array}{lrrr}\text { TS10 } & & & \\ \text { C } & 1.3501034859 & 0.0594677858 & -0.2100318140 \\ \text { C } & 0.0944362290 & 0.4807244278 & 0.5693207711 \\ \text { O } & 0.2374568693 & 0.4287691005 & 1.8829556573 \\ \text { O } & -0.8842042295 & -1.1138465564 & 0.1511699438 \\ \text { O } & -0.7484530378 & 1.2907392423 & 0.0426463158 \\ \text { O } & -1.9063217194 & -0.6531782827 & -0.4443083987 \\ \text { O } & 2.3079925200 & -0.3447778789 & 0.3811101914 \\ \text { C } & 1.2365890385 & 0.2060546988 & -1.6988480553 \\ \text { H } & -1.5552201465 & 0.5777098385 & -0.3648413873 \\ \text { H } & 2.1319440526 & -0.1926995917 & -2.1695238915 \\ \text { H } & 1.1101220237 & 1.2623296834 & -1.9471611628 \\ \text { H } & 0.3481514241 & -0.3182008132 & -2.0590648410 \\ \mathrm{H} & -0.5138965400 & 0.8708682857 & 2.2970666912\end{array}$

TS11

$\begin{array}{lrrr}\mathrm{C} & 1.4707690587 & 0.3057668449 & -0.4679090927 \\ \mathrm{C} & 0.1095387090 & 0.5099853726 & 0.2219202520 \\ \mathrm{C} & 0.2211091207 & 0.4592475754 & 1.7247532140 \\ \mathrm{O} & -0.6756531397 & -0.7036511671 & -0.1940656202 \\ \mathrm{O} & -0.4942151950 & 1.6337796062 & -0.2654762995 \\ \mathrm{O} & -1.8782933483 & -0.4129041547 & -0.5394190896 \\ \mathrm{O} & 2.4991915025 & 0.1484530629 & 0.1224717117 \\ \mathrm{O} & 1.3503434004 & 0.3260531694 & -1.7951194231 \\ \mathrm{H} & -1.4140455344 & 1.3955039717 & -0.4730262628 \\ \mathrm{H} & 0.6856378451 & -0.4740863682 & 2.0412035441 \\ \mathrm{H} & -0.7780645268 & 0.5516190480 & 2.1523689153 \\ \mathrm{H} & 0.8416366030 & 1.2921559294 & 2.0550422849 \\ \mathrm{H} & 2.2258295648 & 0.1897712295 & -2.1796360340\end{array}$

TS12

$\begin{array}{lrrr}\mathrm{C} & 1.4944973492 & 0.2261240643 & -0.3778242613 \\ \mathrm{C} & 0.1495930808 & 0.3776590922 & 0.3532919387 \\ \mathrm{C} & 0.2757789288 & 0.3280195601 & 1.8545453479 \\ \mathrm{O} & -0.5954197795 & -0.8355613429 & -0.0887500648 \\ \mathrm{O} & -0.4800590108 & 1.4918558270 & -0.1341501622 \\ \mathrm{O} & -1.8466095091 & -0.7199414226 & 0.2113904765 \\ \mathrm{O} & 2.5328548266 & 0.1673564401 & 0.1960903620 \\ \mathrm{O} & 1.3629569268 & 0.2161930568 & -1.7289770458 \\ \mathrm{H} & -1.3427882238 & 1.5639114411 & 0.2900227086 \\ \mathrm{H} & 0.7886721132 & -0.5822542948 & 2.1632397589 \\ \mathrm{H} & -0.7226726212 & 0.3552933108 & 2.2956249950 \\ \mathrm{H} & 0.8547851194 & 1.1892458109 & 2.1862897695 \\ \mathrm{H} & 1.2774947996 & -0.6753975430 & -2.0817688230\end{array}$




$\begin{array}{lrrc}\text { TS13 } & & & \\ \text { C } & -1.05667889 & -0.82655638 & -0.11163333 \\ \text { H } & -0.74220216 & -1.35688424 & -1.01086438 \\ \text { C } & -0.08732000 & 0.28096300 & 0.15449496 \\ \text { O } & 1.10899460 & -0.16508716 & 0.64947510 \\ \text { O } & 2.01032996 & 0.86081785 & 0.67610264 \\ \text { O } & 0.02415575 & 1.19679189 & -0.83457887 \\ \text { H } & 0.89935321 & 1.61146700 & -0.72905689 \\ \text { C } & -0.65477967 & 1.21122003 & 1.55157351 \\ \text { O } & 0.30119452 & 1.92740536 & 1.99343896 \\ \text { O } & -1.79733539 & 1.08581817 & 1.82999420 \\ \text { H } & 1.22913623 & 1.62042820 & 1.50866151 \\ \text { H } & -2.04464149 & -0.39387771 & -0.25824049 \\ \text { H } & -1.08397317 & -1.50452197 & 0.74025333\end{array}$

\section{TS14}

$\mathrm{H}$

C

$\mathrm{O}$

$\mathrm{O}$

$\mathrm{O}$

$\mathrm{H}$

C

$\mathrm{O}$

$\mathrm{O}$

$\mathrm{H}$

$\mathrm{H}$

$\mathrm{H}$

$-1.0230530308-0.8545611584-0.1147809308$

$-0.7064172264-1.3692075450-1.0227969049$

$\begin{array}{lll}-0.0829436012 & 0.2858229042 & 0.1578804821\end{array}$

$\begin{array}{llll}1.1173169578 & -0.1871459740 & 0.6114428054\end{array}$

$\begin{array}{llll}2.0167096697 & 0.7997754271 & 0.8017399718\end{array}$

$\begin{array}{llll}0.0926255744 & 1.1943848047 & -0.8637500019\end{array}$

$\begin{array}{llll}-0.7642356069 & 1.4202604818 & -1.2384678414\end{array}$

$\begin{array}{llll}-0.6642420202 & 1.2565718106 & 1.4596712273\end{array}$

$\begin{array}{llll}0.2854364974 & 1.9264315595 & 1.9468624424\end{array}$

$\begin{array}{llll}-1.8442293008 & 1.2094968561 & 1.6300517392\end{array}$

$\begin{array}{lll}1.2864805931 & 1.5585942934 & 1.4189065290\end{array}$

$\begin{array}{llll}-2.0321347491 & -0.4594856815 & -0.2298155315\end{array}$

$\begin{array}{llll}-1.0191541170 & -1.5451591684 & 0.7277247132\end{array}$

TS15

C $\quad-1.0014888991 \quad-0.6971230074 \quad-0.3035393184$

$\mathrm{H} \quad-0.8383577721 \quad-1.6931414743 \quad-0.7332369190$

$\begin{array}{lllll}\text { C } & 0.2157890116 & 0.1402037598 & -0.4203021058\end{array}$

$\begin{array}{lllll}\mathrm{O} & 1.1882846786 & -0.0269968940 & 0.4742095338\end{array}$

$\begin{array}{lllll}\mathrm{O} & & 1.8351372293 & 1.3050569457 & 0.7118433624\end{array}$

$\begin{array}{llllll}\text { O } & 0.5809907126 & 0.5533408399 & -1.6589633578\end{array}$

$\mathrm{H} \quad \begin{array}{lllll}1.3853840898 & 1.0778805371 & -1.5679724444\end{array}$

$\mathrm{H} \quad \begin{array}{llll}1.2502488467 & 1.6663300405 & 1.3904313358\end{array}$

$\mathrm{H} \quad-\quad-1.8264602945 \quad-0.2306070339-0.8439978815$

$\mathrm{H} \quad-1.2702669631 \quad-0.8127930032 \quad 0.7455865051$

$\begin{array}{llll}\text { TS16 } & & & \\ \text { C } & -1.0104649649 & -0.7274755585 & -0.2628296478 \\ \text { H } & -0.8641254765 & -1.7813712402 & -0.5317455310 \\ \text { C } & 0.2324895991 & 0.0626414133 & -0.4855147517 \\ \text { O } & 1.1753187735 & 0.0183938540 & 0.4454977645 \\ \text { O } & 1.7072157148 & 1.3865271226 & 0.6752128041 \\ \text { O } & 0.7077435708 & 0.2576599479 & -1.7467928305 \\ \text { H } & -0.0220016938 & 0.3982410779 & -2.3538760811\end{array}$ 


$\begin{array}{lrrc}\mathrm{H} & 1.3118154500 & 1.5709435886 & 1.5358951907 \\ \mathrm{H} & -1.8351947126 & -0.3404463333 & -0.8675628820 \\ \mathrm{H} & -1.2960296804 & -0.6757175023 & 0.7867709347\end{array}$

Table S.2 DFT energetics of reactants and intermediate structures for the $\mathrm{PA}+\mathrm{HO}_{2}$ reactions calculated using M11/cc-pVTZ structures. These singlepoint energies were calculated from M11/cc-pVXZ where $\mathrm{X}=\mathrm{D}, \mathrm{T}, \mathrm{Q}$ as well as the CBS energies determined from a power fit.

\begin{tabular}{lcccc}
\hline \hline M11/cc-pVXZ & cc-pVDZ & cc-pVTZ & cc-pVQZ & CBS \\
\hline Pyruvic acid Tc & -342.277100 & -342.410034 & -342.438281 & -342.463056 \\
Pyruvic acid Tt & -342.272514 & -342.405301 & -342.433291 & -342.458102 \\
Pyruvic acid Cc & -342.257523 & -342.391865 & -342.420282 & -342.445356 \\
Pyruvic acid Ct & -342.269680 & -342.402505 & -342.430482 & -342.455306 \\
HO $_{2}$ & -150.856648 & -150.922499 & -150.934018 & -150.946989 \\
PRC1 & -493.156069 & -493.349751 & -493.388931 & -493.425585 \\
PRC2 & -493.151309 & -493.345759 & -493.384517 & -493.421480 \\
PRC3 & -493.162078 & -493.353157 & -493.391481 & -493.427736 \\
PRC4 & -493.148891 & -493.343118 & -493.381694 & -493.418653 \\
INT1 & -342.258237 & -342.392802 & -342.421519 & -342.446562 \\
INT2 & -342.247125 & -342.383175 & -342.412330 & -342.437616 \\
INT3 & -493.088139 & -493.286515 & -493.323879 & -493.362202 \\
INT4 & -493.101494 & -493.298304 & -493.336017 & -493.373856 \\
INT5 & -493.115272 & -493.309945 & -493.348610 & -493.385654 \\
INT6 ${ }^{a}$ & -493.159479 & -493.351397 & -493.390589 & -493.426805 \\
INT7 & -493.162286 & -493.354151 & -493.392961 & -493.429272 \\
INT8 & -493.157371 & -493.349662 & -493.388575 & -493.424962 \\
INT9 & -493.161786 & -493.360790 & -493.399692 & -493.437736 \\
INT10 & -304.625417 & -304.751351 & -304.777189 & -304.800920 \\
\hline \hline
\end{tabular}


Table S.3 DFT energetics of transition state structures of $\mathrm{PA}+\mathrm{HO}_{2}$ reactions calculated using M11/cc-pVTZ structures. These were calculated from M11/cc-pVXZ where X=D,T,Q as well as the CBS energies determined from a power fit.

\begin{tabular}{lcccc}
\hline \hline M11/cc-pVXZ & cc-pVDZ & $c c-p V T Z$ & $c c-p V Q Z$ & CBS \\
\hline TS1 & -493.101401 & -493.297078 & -493.336380 & -493.373491 \\
TS2 & -493.090756 & -493.286108 & -493.324804 & -493.362006 \\
TS3 & -493.102138 & -493.291872 & -493.330100 & -493.366050 \\
TS4 & -493.083829 & -493.278062 & -493.317261 & -493.354046 \\
TS5 & -493.089043 & -493.284925 & -493.322899 & -493.360435 \\
TS6 & -493.114273 & -493.309076 & -493.347772 & -493.384839 \\
TS7 & -493.143133 & -493.332932 & -493.371351 & -493.407263 \\
TS8 & -493.142158 & -493.331899 & -493.369951 & -493.405952 \\
TS9 & -493.139563 & -493.329054 & -493.367285 & -493.403175 \\
TS10 & -493.132203 & -493.321870 & -493.359870 & -493.395868 \\
TS11 & -493.156360 & -493.348414 & -493.387269 & -493.423613 \\
TS12 & -493.138260 & -493.331932 & -493.371136 & -493.407781 \\
TS13 & -493.129799 & -493.320799 & -493.359782 & -493.395831 \\
TS14 & -493.090415 & -493.281772 & -493.321081 & -493.357126 \\
TS15 & -304.622443 & -304.748980 & -304.774735 & -304.798637 \\
TS16 & -304.649424 & -304.751225 & -304.772877 & -304.791844 \\
\hline \hline
\end{tabular}

Table S.4 DFT energetics of products produced from the reactions between $\mathrm{PA}+\mathrm{HO}_{2}$ calculated using M11/cc-pVTZ structures. These were calculated from M11/cc-pVXZ where X=D,T,Q as well as the CBS energies determined from a power fit.

\begin{tabular}{lcccc}
\hline \hline $\mathrm{M} 11 / \mathrm{cc}-\mathrm{pVXZ}$ & $\mathrm{cc}-\mathrm{pVDZ}$ & $\mathrm{cc}-\mathrm{pVTZ}$ & $\mathrm{cc}-\mathrm{pVQZ}$ & $\mathrm{CBS}$ \\
\hline $\mathrm{OH}$ & -75.700975 & -75.7383954 & -75.7452469 & -75.7525314 \\
$\mathrm{CO}_{2}$ & -188.52534 & -188.601646 & -188.615491 & -188.630381 \\
$\mathrm{H}_{2} \mathrm{O}_{2}$ & -151.495316 & -151.567704 & -151.581733 & -151.595606 \\
$\mathrm{P} 1$ & -341.616882 & -341.747445 & -341.774970 & -341.799365 \\
$\mathrm{P} 2$ & -153.105008 & -153.166369 & -153.178659 & -153.190306 \\
$\mathrm{P} 3 / \mathrm{P} 4$ & -153.673339 & -153.736279 & -153.750208 & -153.761781 \\
$\mathrm{P} 5$ & -342.229370 & -342.364028 & -342.392012 & -342.417285 \\
$\mathrm{P} 6$ & -228.994860 & -229.088824 & -229.108566 & -229.126141 \\
\hline \hline
\end{tabular}


Table $\mathrm{S} .5 \mathrm{Ab}$ initio energetics of reactants and transition state structures of $\mathrm{PA}+\mathrm{HO}_{2}$ reactions calculated using M11/cc-pVTZ structures. These were calculated using structures obtain using M11/cc-pVTZ with CCSD(T)/cc-pVXZ where X=D,T,Q. The CBS energies were then determined from a power fit.

\begin{tabular}{lcccc}
\hline \hline M11/cc-pVXZ & cc-pVDZ & $c c-p V T Z$ & $c c-p V Q Z$ & CBS \\
\hline HO2 & -150.557770 & -150.712067 & -150.759486 & -150.784109 \\
pyruvic Tc & -341.563309 & -341.905005 & -342.010520 & -342.064906 \\
pyruvic Tt & -341.559959 & -341.900760 & -342.006104 & -342.060318 \\
pyruvic Cc & -341.545478 & -341.887704 & -341.993363 & -342.047839 \\
pyruvic Ct & -341.557487 & -341.898103 & -342.003504 & -342.057657 \\
TS1 & -492.084092 & -492.582804 & -492.735629 & -492.815339 \\
TS2 & -492.066527 & -492.564822 & -492.717747 & -492.797326 \\
TS3 & -492.076089 & -492.573033 & -492.725323 & -492.804749 \\
TS4 & -492.057711 & -492.558929 & -492.712270 & -492.792452 \\
TS5 & -492.075665 & -492.567241 & -492.719054 & -492.797292 \\
TS6 & -492.096044 & -492.594516 & -492.747096 & -492.826816 \\
TS7 & -492.122341 & -492.619250 & -492.771389 & -492.850849 \\
TS8 & -492.121556 & -492.617428 & -492.769564 & -492.848769 \\
TS9 & -492.117211 & -492.613734 & -492.766282 & -492.845531 \\
TS10 & -492.110982 & -492.607171 & -492.759500 & -492.838728 \\
TS11 & -492.135262 & -492.633920 & -492.786546 & -492.866299 \\
TS12 & -492.117145 & -492.616645 & -492.769565 & -492.849442 \\
TS13 & -492.103175 & -492.604282 & -492.757169 & -492.837451 \\
TS14 & -492.091063 & -492.593094 & -492.746168 & -492.826626 \\
TS15 & -303.988377 & -304.307665 & -304.403557 & -304.455140 \\
TS16 & -303.980896 & -304.300774 & -304.396804 & 304.448494 \\
\hline \hline & & & &
\end{tabular}

Table S.6 Harmonic frequncies of $\mathrm{PA}+\mathrm{HO}_{2}$ reactants and intermediates determined from the M11/cc-pVTZ stationary point structures. Frequencies are in $\mathrm{cm}^{-1}$.

\begin{tabular}{ccccccccc}
\hline \hline $\mathrm{HO}_{2}$ & 1247.19 & 1449.23 & 3691.67 & & & & & \\
\hline \multirow{3}{*}{ Pyruvic Tc } & 100.98 & 130.19 & 260.17 & 397.57 & 400.14 & 532.55 & 613.37 & 706.68 \\
& 743.56 & 783.18 & 979.02 & 1038.68 & 1171.91 & 1251.40 & 1374.91 & 1415.53 \\
& 1443.33 & 1445.87 & 1839.00 & 1893.35 & 3062.93 & 3131.92 & 3188.57 & 3685.08 \\
\hline \multirow{3}{*}{ Pyruvic Tt } & 55.04 & 143.46 & 251.14 & 380.39 & 388.40 & 521.77 & 598.07 & 620.69 \\
& 744.79 & 751.80 & 972.29 & 1041.44 & 1153.83 & 1221.47 & 1372.59 & 1413.77 \\
& 1447.04 & 1450.40 & 1846.65 & 1868.14 & 3062.98 & 3133.90 & 3186.57 & 3793.07 \\
\hline \multirow{2}{*}{ Pyruvic Cc } & 28.86 & 221.20 & 276.69 & 368.73 & 405.50 & 481.44 & 485.88 & 617.98 \\
& 714.55 & 760.56 & 968.30 & 1032.32 & 1135.47 & 1186.54 & 1309.29 & 1369.15 \\
& 1464.41 & 1473.38 & 1874.28 & 1910.58 & 3038.04 & 3109.79 & 3176.92 & 3868.32 \\
\hline Pyruvic Ct & 41.03 & 168.88 & 259.25 & 386.24 & 399.90 & 486.63 & 615.71 & 619.39
\end{tabular}




\begin{tabular}{|c|c|c|c|c|c|c|c|c|}
\hline & 742.38 & 743.53 & 978.51 & 1041.91 & 1150.62 & 1193.66 & 1351.03 & 1379.23 \\
\hline & 1450.62 & 1454.78 & 1870.97 & 1884.12 & 3060.90 & 3132.82 & 3183.18 & 3788.30 \\
\hline \multirow{5}{*}{ PRC1 } & 48.36 & 58.66 & 111.50 & 131.43 & 136.69 & 182.07 & 246.74 & 276.84 \\
\hline & 399.24 & 403.53 & 536.57 & 594.86 & 624.47 & 722.19 & 802.56 & 832.75 \\
\hline & 982.75 & 1035.19 & 1189.87 & 1251.51 & 1303.30 & 1370.59 & 1410.51 & 1440.00 \\
\hline & 1442.91 & 1512.22 & 1829.49 & 1871.88 & 3064.09 & 3132.98 & 3190.32 & 3445.38 \\
\hline & 3538.77 & & & & & & & \\
\hline \multirow{5}{*}{ PRC2 } & 26.77 & 44.99 & 85.42 & 91.01 & 116.56 & 132.92 & 186.85 & 280.03 \\
\hline & 401.41 & 412.58 & 542.96 & 570.58 & 622.38 & 680.28 & 738.31 & 786.50 \\
\hline & 987.73 & 1041.62 & 1180.34 & 1254.92 & 1280.77 & 1375.76 & 1412.88 & 1440.97 \\
\hline & 1450.34 & 1540.55 & 1809.50 & 1897.97 & 3055.81 & 3124.53 & 3183.37 & 3501.58 \\
\hline & 3716.96 & & & & & & & \\
\hline \multirow{5}{*}{ PRC3 } & 46.89 & 71.14 & 108.38 & 128.51 & 141.03 & 191.70 & 269.63 & 334.43 \\
\hline & 405.14 & 412.37 & 542.42 & 620.99 & 718.74 & 802.64 & 806.74 & 950.72 \\
\hline & 977.74 & 1043.48 & 1183.80 & 1316.56 & 1348.74 & 1383.42 & 1446.44 & 1450.71 \\
\hline & 1494.76 & 1600.92 & 1767.92 & 1872.11 & 3062.68 & 3090.60 & 3133.42 & 3187.22 \\
\hline & 3264.79 & & & & & & & \\
\hline \multirow{5}{*}{ PRC4 } & 30.23 & 41.38 & 61.06 & 82.88 & 119.86 & 141.44 & 190.34 & 279.25 \\
\hline & 387.42 & 404.54 & 534.53 & 607.35 & 610.97 & 624.72 & 747.23 & 759.29 \\
\hline & 984.22 & 1046.00 & 1163.95 & 1231.24 & 1283.93 & 1377.95 & 1419.48 & 1443.89 \\
\hline & 1455.72 & 1546.03 & 1838.74 & 1856.90 & 3057.20 & 3127.52 & 3182.18 & 3456.95 \\
\hline & 3790.56 & & & & & & & \\
\hline \multirow{3}{*}{ INT1 } & 70.09 & 282.28 & 371.04 & 411.98 & 429.30 & 532.27 & 587.90 & 636.75 \\
\hline & 745.39 & 799.83 & 825.87 & 921.99 & 981.56 & 1163.92 & 1182.70 & 1322.61 \\
\hline & 1416.62 & 1453.88 & 1739.81 & 1843.82 & 3171.28 & 3283.00 & 3801.39 & 3858.37 \\
\hline \multirow{3}{*}{ INT2 } & 84.05 & 281.70 & 351.63 & 410.41 & 423.67 & 540.17 & 558.61 & 626.96 \\
\hline & 738.18 & 805.87 & 815.19 & 887.31 & 993.46 & 1143.63 & 1192.92 & 1297.89 \\
\hline & 1425.33 & 1445.71 & 1720.36 & 1900.19 & 3167.91 & 3264.69 & 3845.65 & 3854.04 \\
\hline \multirow{5}{*}{ INT3 } & 22.41 & 39.01 & 70.74 & 83.46 & 96.94 & 99.71 & 101.46 & 128.09 \\
\hline & 176.53 & 251.19 & 318.42 & 556.44 & 671.56 & 686.67 & 726.16 & 918.23 \\
\hline & 986.61 & 1017.41 & 1100.83 & 1302.81 & 1348.89 & 1380.70 & 1399.84 & 1407.13 \\
\hline & 1426.52 & 1493.14 & 1649.88 & 2436.34 & 2580.92 & 2996.37 & 3097.73 & 3128.74 \\
\hline & 3594.53 & & & & & & & \\
\hline \multirow{3}{*}{ INT4 } & 81.21 & 131.08 & 190.55 & 202.46 & 232.02 & 353.64 & 559.23 & 847.95 \\
\hline & 942.41 & 1074.94 & 1117.19 & 1134.02 & 1306.24 & 1356.17 & 1399.71 & 1441.45 \\
\hline & 1471.12 & 1489.81 & 1684.41 & 2151.65 & 3036.41 & 3112.36 & 3140.28 & 3287.02 \\
\hline \multirow{4}{*}{ INT5 } & 44.13 & 84.47 & 126.02 & 157.04 & 169.66 & 220.97 & 267.90 & 308.79 \\
\hline & 365.97 & 394.59 & 521.89 & 617.32 & 656.26 & 706.21 & 745.93 & 833.63 \\
\hline & 1008.98 & 1049.98 & 1192.94 & 1302.25 & 1320.07 & 1369.63 & 1407.90 & 1458.73 \\
\hline & 1469.72 & 1486.20 & 1574.63 & 1949.14 & 3070.12 & 3150.30 & 3180.58 & 3270.67 \\
\hline & & & & SI & & & & \\
\hline
\end{tabular}




\begin{tabular}{|c|c|c|c|c|c|c|c|c|}
\hline & 3619.70 & & & & & & & \\
\hline \multirow{5}{*}{ INT6 } & 38.50 & 133.83 & 187.71 & 225.62 & 259.80 & 310.82 & 375.54 & 392.52 \\
\hline & 465.62 & 496.24 & 565.17 & 595.32 & 655.96 & 747.88 & 782.89 & 833.56 \\
\hline & 975.40 & 1084.10 & 1134.55 & 1182.20 & 1269.09 & 1296.58 & 1372.14 & 1389.03 \\
\hline & 1453.59 & 1468.98 & 1474.62 & 1873.01 & 3078.68 & 3170.90 & 3179.82 & 3791.59 \\
\hline & 3793.66 & & & & & & & \\
\hline \multirow{5}{*}{ INT7 } & 41.33 & 113.09 & 196.57 & 243.82 & 252.23 & 305.96 & 366.13 & 380.93 \\
\hline & 405.90 & 460.36 & 563.89 & 589.29 & 678.84 & 734.84 & 806.21 & 848.24 \\
\hline & 976.00 & 1097.52 & 1127.99 & 1189.27 & 1265.32 & 1292.72 & 1332.54 & 1398.74 \\
\hline & 1442.64 & 1469.22 & 1497.63 & 1870.14 & 3068.12 & 3160.38 & 3172.16 & 3794.64 \\
\hline & 3803.63 & & & & & & & \\
\hline \multirow{5}{*}{ INT8 } & 49.54 & 111.46 & 195.78 & 245.97 & 275.91 & 339.02 & 368.88 & 396.01 \\
\hline & 424.58 & 506.95 & 564.64 & 655.70 & 698.14 & 725.56 & 799.74 & 842.92 \\
\hline & 972.16 & 1088.32 & 1137.99 & 1206.8 & 1254.16 & 1293.74 & 1352.54 & 1394.68 \\
\hline & 1420.96 & 1468.73 & 1488.68 & 1904.01 & 3065.29 & 3154.79 & 3174.69 & 3746.56 \\
\hline & 3780.73 & & & & & & & \\
\hline \multirow{5}{*}{ INT9 } & 34.46 & 54.83 & 84.96 & 108.85 & 145.24 & 165.60 & 213.76 & 306.29 \\
\hline & 374.59 & 399.30 & 492.92 & 526.76 & 618.24 & 674.90 & 680.63 & 866.98 \\
\hline & 956.55 & 1020.57 & 1071.19 & 1108.53 & 1301.21 & 1358.78 & 1398.58 & 1418.43 \\
\hline & 1422.00 & 1461.92 & 1474.97 & 2436.85 & 3034.30 & 3128.92 & 3173.53 & 3746.87 \\
\hline & 3795.51 & & & & & & & \\
\hline \multirow{3}{*}{ INT10 } & 136.16 & 157.37 & 267.90 & 305.01 & 372.30 & 455.42 & 527.65 & 607.12 \\
\hline & 870.66 & 953.48 & 989.02 & 1065.49 & 1112.85 & 1292.60 & 1358.00 & 1383.42 \\
\hline & 1419.98 & 1459.22 & 1474.45 & 3030.12 & 3123.44 & 3166.36 & 3779.60 & 3808.01 \\
\hline
\end{tabular}

Table S.7 Harmonic frequencies of $\mathrm{PA}+\mathrm{HO}_{2}$ transition state structures determined from the M11/cc-pVTZ stationary point structures. Frequencies are in cm-1.

\begin{tabular}{rrrrrrrrr}
\hline \hline & 27.33 & 70.95 & 84.95 & 132.93 & 248.14 & 276.87 & 402.07 & 415.29 \\
TS1 & 472.32 & 515.72 & 535.91 & 609.08 & 625.43 & 705.58 & 789.67 & 816.62 \\
& 995.55 & 1016.40 & 1061.81 & 1112.54 & 1187.12 & 1261.58 & 1400.56 & 1415.41 \\
& 1440.32 & 1454.20 & 1744.23 & 1892.98 & 3148.13 & 3262.10 & 3692.01 & 3747.27 \\
& $2334.24 i$ & & & & & & & \\
\hline & 39.27 & 55.40 & 91.52 & 154.85 & 189.41 & 203.10 & 248.58 & 278.01 \\
TS2 & 320.68 & 461.80 & 513.44 & 575.02 & 641.36 & 740.60 & 816.40 & 959.86 \\
& 1022.84 & 1129.84 & 1204.58 & 1222.94 & 1352.01 & 1443.02 & 1449.24 & 1471.23 \\
& 1577.65 & 1651.82 & 1823.82 & 1953.95 & 3065.94 & 3150.71 & 3187.76 & 3362.41 \\
\hline TS3 & $4896.87 i$ & & & & & & & \\
\hline
\end{tabular}




\begin{tabular}{|c|c|c|c|c|c|c|c|c|}
\hline & 536.75 & 601.35 & 635.96 & 653.02 & 726.37 & 776.95 & 832.49 & 937.17 \\
\hline & 1044.57 & 1171.49 & 1198.31 & 1279.25 & 1290.17 & 1331.48 & 1376.08 & 1468.12 \\
\hline & 1483.16 & 1640.10 & 1672.87 & 1826.14 & 1862.13 & 3108.15 & 3249.99 & 3791.69 \\
\hline & $1556.98 i$ & & & & & & & \\
\hline \multirow{5}{*}{ TS4 } & 29.97 & 73.95 & 108.03 & 188.56 & 257.49 & 262.19 & 368.57 & 455.77 \\
\hline & 550.84 & 597.41 & 637.02 & 673.45 & 713.91 & 810.46 & 875.49 & 942.20 \\
\hline & 1035.66 & 1161.03 & 1185.97 & 1301.79 & 1333.93 & 1373.96 & 1399.47 & 1441.95 \\
\hline & 1491.04 & 1520.24 & 1646.08 & 1681.80 & 1846.22 & 3109.16 & 3204.41 & 3757.48 \\
\hline & $1110.95 i$ & & & & & & & \\
\hline \multirow{5}{*}{ TS5 } & 39.87 & 40.67 & 92.07 & 96.31 & 119.29 & 161.26 & 184.44 & 199.54 \\
\hline & 255.30 & 293.07 & 532.70 & 550.13 & 587.26 & 680.85 & 851.50 & 932.89 \\
\hline & 1075.28 & 1113.69 & 1322.15 & 1345.42 & 1352.39 & 1403.05 & 1435.30 & 1473.64 \\
\hline & 1500.64 & 1535.55 & 2329.71 & 3032.86 & 3106.33 & 3138.31 & 3363.49 & 3465.34 \\
\hline & $210.40 i$ & & & & & & & \\
\hline \multirow{5}{*}{ TS6 } & 47.90 & 96.54 & 142.47 & 161.87 & 184.95 & 238.36 & 308.24 & 351.61 \\
\hline & 381.67 & 492.19 & 612.93 & 742.49 & 763.11 & 857.15 & 902.83 & 999.38 \\
\hline & 1046.01 & 1205.92 & 1332.40 & 1377.40 & 1389.61 & 1438.58 & 1468.63 & 1494.39 \\
\hline & 1525.71 & 1605.46 & 1876.66 & 3037.70 & 3065.45 & 3141.80 & 3192.27 & 3301.75 \\
\hline & $174.68 i$ & & & & & & & \\
\hline \multirow{5}{*}{ TS7 } & 63.24 & 102.27 & 171.46 & 218.41 & 237.83 & 284.60 & 386.58 & 468.84 \\
\hline & 534.60 & 589.20 & 623.78 & 667.52 & 716.35 & 778.53 & 799.83 & 995.11 \\
\hline & 1027.60 & 1063.76 & 1155.19 & 1231.83 & 1373.58 & 1398.27 & 1417.69 & 1455.58 \\
\hline & 1458.08 & 1642.88 & 1857.82 & 1908.21 & 3065.13 & 3146.83 & 3181.03 & 3797.68 \\
\hline & $925.78 i$ & & & & & & & \\
\hline \multirow{5}{*}{ TS8 } & 78.01 & 109.55 & 179.97 & 213.68 & 255.16 & 298.57 & 393.15 & 509.75 \\
\hline & 543.44 & 628.96 & 680.44 & 694.24 & 737.47 & 773.51 & 818.37 & 997.32 \\
\hline & 1061.68 & 1073.94 & 1170.83 & 1253.77 & 1373.41 & 1395.89 & 1409.45 & 1456.05 \\
\hline & 1460.43 & 1613.09 & 1896.86 & 1928.53 & 3063.44 & 3146.11 & 3181.14 & 3706.11 \\
\hline & $832.42 i$ & & & & & & & \\
\hline \multirow{5}{*}{ TS9 } & 96.70 & 102.89 & 138.34 & 197.38 & 249.91 & 300.81 & 397.74 & 492.96 \\
\hline & 520.00 & 568.22 & 615.58 & 682.83 & 688.59 & 821.38 & 940.65 & 977.93 \\
\hline & 1009.74 & 1054.46 & 1181.75 & 1255.94 & 1364.67 & 1383.82 & 1436.80 & 1446.86 \\
\hline & 1454.57 & 1644.52 & 1840.40 & 1930.91 & 3061.21 & 3131.98 & 3187.14 & 3671.92 \\
\hline & $804.85 i$ & & & & & & & \\
\hline \multirow{5}{*}{ TS10 } & 72.90 & 112.47 & 144.20 & 206.62 & 246.98 & 299.36 & 385.91 & 447.60 \\
\hline & 511.77 & 552.02 & 580.36 & 611.42 & 684.34 & 793.59 & 922.00 & 972.80 \\
\hline & 1038.90 & 1057.02 & 1152.27 & 1216.35 & 1366.27 & 1379.78 & 1443.41 & 1451.55 \\
\hline & 1459.83 & 1593.18 & 1870.65 & 1916.61 & 3062.11 & 3134.35 & 3185.37 & 3806.96 \\
\hline & $853.317 i$ & & & & & & & \\
\hline TS11 & 42.70 & 214.32 & 235.96 & 252.31 & 327.82 & 364.24 & 383.51 & 406.24 \\
\hline
\end{tabular}




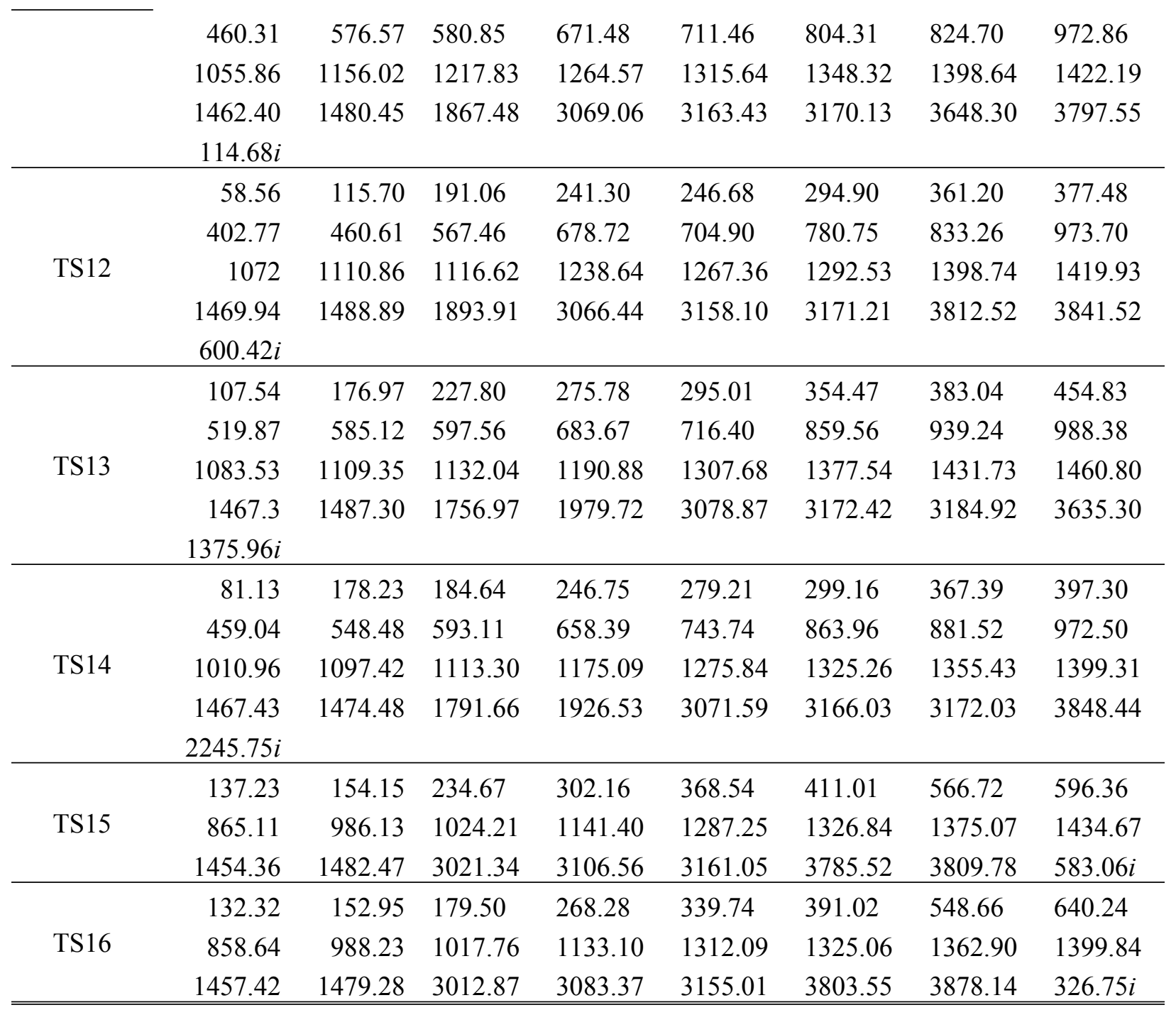

Table S.7 Harmonic Frequncies of the products produced from the reactions between $\mathrm{PA}+\mathrm{HO}_{2}$, determined from the M11/cc-pVTZ stationary point structures. Frequencies are in $\mathrm{cm}^{-1}$.

\begin{tabular}{crrrrrrrr}
\hline \hline $\mathrm{OH}$ & 3767.36 & \multicolumn{7}{c}{} \\
\hline $\mathrm{H}_{2} \mathrm{O}_{2}$ & 380.94 & 1034.80 & 1349.62 & 1460.53 & 3816.78 & 3818.58 & & \\
\hline $\mathrm{CO}_{2}$ & 684.26 & 686.82 & 1401.86 & 2439.26 & & & & \\
\hline & 109.27 & 276.86 & 379.62 & 404.80 & 456.64 & 537.56 & 612.56 & 724.72 \\
$\mathrm{P} 1$ & 765.64 & 792.83 & 878.91 & 1011.02 & 1192.00 & 1281.50 & 1405.76 & 1466.44 \\
& 1688.77 & 1889.47 & 3179.12 & 3306.66 & 3675.63 & & & \\
\hline $\mathrm{P} 2$ & 100.47 & 470.08 & 859.01 & 946.47 & 1047.41 & 1337.95 & 1446.80 & 1449.02 \\
& 1985.70 & 3053.72 & 3155.95 & 3156.85 & & & & \\
\hline \multirow{2}{*}{$\mathrm{P} 3 / \mathrm{P} 4$} & 56.23 & 519.19 & 738.22 & 918.89 & 969.13 & 1064.52 & 1323.49 & 1330.13 \\
& 1368.59 & 1438.41 & 1463.64 & 3022.83 & 3109.50 & 3120.68 & 3804.16 & \\
\hline \multirow{2}{*}{$\mathrm{P} 5$} & 169.28 & 176.54 & 310.22 & 354.43 & 390.17 & 448.88 & 584.49 & 629.74 \\
& 720.13 & 808.25 & 1006.48 & 1040.86 & 1152.63 & 1218.77 & 1318.46 & 1378.70
\end{tabular}




\begin{tabular}{rrrrrrrrr} 
& 1426.28 & 1468.63 & 1480.67 & 2019.57 & 3069.38 & 3151.89 & 3176.27 & 3843.13 \\
\hline \multirow{4}{*}{ P6 } & 78.32 & 421.03 & 544.34 & 592.64 & 670.78 & 872.41 & 998.17 & 1069.67 \\
& 1210.25 & 1336.29 & 1404.08 & 1462.15 & 1468.11 & 1858.71 & 3074.03 & 3150.32 \\
& 3193.13 & 3799.32 & & & & & & \\
\hline \hline
\end{tabular}

